\title{
Observation of Astrophysical Neutrinos in Six Years of IceCube Data
}

\author{
The IceCube Collaboration ${ }^{\dagger *}$ \\ $\dagger$ http://icecube.wisc.edu/collaboration/authors/icrc17_icecube \\ E-mail: analysis@icecube.wisc.edu
}

\begin{abstract}
Cosmic rays arriving at Earth include the most energetic particles ever observed. The mechanism of their acceleration and their sources are, however, still mostly unknown. Observing astrophysical neutrinos can help solve this problem. Because neutrinos are produced in hadronic interactions and are neither absorbed nor deflected, they will point directly back to their source. This contribution covers continued studies of the high-energy astrophysical neutrino flux observed at the IceCube neutrino observatory, extending them from four to six years of data with a focus on energies above $60 \mathrm{TeV}$. The spectrum and spatial clustering of the observed neutrinos are discussed.
\end{abstract}

35th International Cosmic Ray Conference - ICRC2017

10-20 July, 2017

Bexco, Busan, Korea

${ }^{*}$ Presenter: C. Kopper, University of Alberta 


\section{Introduction}

Observation of high-energy neutrinos provides insight into the problem of the origin and acceleration mechanism of high-energy cosmic rays. Cosmic ray protons and nuclei interacting with gas and photons present in the environment of sources and in the interstellar and intergalactic space produce neutrinos through decay of charged pions and kaons. These neutrinos have energies related to the cosmic rays that produced them and point back to their sources since neutrinos are neither affected by magnetic fields nor absorbed by matter opaque to radiation. Large-volume Cherenkov detectors like IceCube [1] observe these neutrinos when they interact in or around the instrumented volume by measuring the light produced by charged particles created in the interaction.

Here we present an update to the IceCube high-energy search for events with interaction vertices inside the detector fiducial volume. This search, previously performed on two [2], three [3] and four [4] years of detector data, led to the discovery of an astrophysical neutrino flux above atmospheric backgrounds [3]. This update extends the data-taking period by two more years to six years from early 2010 to early 2016 for a total livetime of 2078 days.

\section{Event Selection}

Neutrinos in IceCube are detected by observing the Cherenkov light in the glacial ice from secondary particles created by the interaction of high-energy neutrinos. We observe two main event classes: track-like events from charged-current interactions of muon neutrinos (and from a minority of tau neutrino interactions) and shower-like events from all other interactions (neutral-current interactions and charged-current interactions of electron and tau neutrinos). Note that tau neutrino interactions at the highest energies (above a few hundred $\mathrm{TeV}$ ) can lead to different event shapes such as two cascades separated by a short tau track, which are included in the simulation but are not explicitly reconstructed beyond a classification into tracks and cascades ${ }^{1}$. We determine the event direction and deposited energy in the detector based on the time sequence of the Cherenkov photons and total recorded light. Energy is reconstructed as electromagnetic-equivalent energy (the energy deposited by the events assuming all light was created in electromagnetic showers). Although deposited energy resolution is similar for all events, shower-like events have a typical directional resolution of around 15 degrees, mostly dominated by the uncertainty in the modelling of the glacial ice, whereas track-like events have resolutions of much better than 1 degree.

In the analysis presented here ("high-energy starting events (HESE)"), signal events are selected by requiring the neutrino interaction vertex to be located inside the IceCube fiducial detector volume. We achieve this by means of a simple anti-coincidence veto method [2], requiring that fewer than 3 of the first 250 detected photoelectrons (p.e.) be on the outer detector boundary. In order to ensure a large enough number of photons are detected, we also require at least 6000 p.e. in total charge for each event, corresponding to a soft threshold of about $30 \mathrm{TeV}$ in deposited energy in the detector.

\section{Atmospheric Backgrounds}

Backgrounds to astrophysical neutrinos are entirely due to cosmic ray air showers. Muons produced in these showers, mainly from $\pi$ and $\mathrm{K}$ decays enter the detector from above. Due to the

\footnotetext{
${ }^{1}$ An analysis using the sample discussed here and adding an explicit tau identifier can be found in [5].
} 
stochasticity of the muon energy loss, in very rare cases muons can pass through the outer veto layer undetected and appear as starting events, especially close to the charge threshold of 6000 p.e. A data-driven method is used to estimate this background by tagging such muons in one layer of the detector and using an equivalent second layer to estimate their passing probability. This procedure yields a total atmospheric muon background of $25.2 \pm 7.3$ events in six years of data.

The same cosmic ray air showers also produce neutrinos from $\pi$ and K decays. The spectrum of these muon-neutrino dominated atmospheric neutrinos is typically one power steeper than the original cosmic ray spectrum. This is due to the increasing lifetime of the parent mesons, making it more and more likely for them to interact before decaying. At energies above around $100 \mathrm{TeV}$, an analogous flux of muons and neutrinos from charm-containing mesons is expected to dominate. The shorter lifetime of these particles allows them to avoid interactions before their decay, leading to a harder spectral slope of this component. Until now, this "prompt" component has not been observed, but limits from data have been placed by previous IceCube analyses [6, 7, 8]. As in previous iterations of this analysis, we use the limit set by an analysis of upgoing muons from muon neutrinos in the 59-string configuration of IceCube [6] for purposes of background estimation. Newer limits are available and will be used in future IceCube analyses of this data sample and extensions of it. Note that some of the down-going atmospheric neutrino background will be vetoed because of accompanying muons from the same air shower. This reduces the background from atmospheric neutrinos in the Southern Sky. This analysis uses the veto probabilities as described in [7]. The total number of expected background events from atmospheric neutrinos in six years of data is $15.6_{-3.9}^{+11.4}$ (accounting for an unknown "prompt" component at or below the upper limit measured in [6]).

\section{Diffuse Flux Fit}

In the full 2078-day sample, we detect 82 events (Fig. 1); 20 of them are observed in the fifth and 8 in the sixth year, respectively (see table 1). Event number 32, observed in the third year and event number 55 in the fifth year were produced by a coincident pair of background muons from unrelated cosmic ray air showers and have been excluded from the analysis.

In order to describe the data, we perform a likelihood fit of all expected components (atmospheric muons, atmospheric neutrinos from $\pi / \mathrm{K}$ decay, atmospheric neutrinos from charm decay and an astrophysical flux assuming a 1:1:1 flavor ratio). The fit is performed in the energy range of $60 \mathrm{TeV}<E_{\mathrm{dep}}<10 \mathrm{PeV}$, which is an extended range compared to [4] to include the Glashow resonance in the prediction. Due to the non-observation of events in this extended energy range the effect on the fit result is negligible. The neutrino interaction model was updated from previous iterations of this analysis by using corrected charged- and neutral-current cross-sections [9], resulting in an approximately $25 \%$ decrease in best-fit normalization.

As in previous iterations of this analysis, we fit an unbroken power-law spectrum with a variable spectral index, $E^{-\gamma}$. The best fit yields a spectral index of $-2.92_{-0.29}^{+0.33}$. We note that all of the events in the recent two years have energies below $200 \mathrm{TeV}$, resulting in a softening of the spectrum compared to previous results [2, 3, 4]. However, due to the large uncertainties these results are still compatible within $2 \sigma$. Furthermore, this result remains compatible with other IceCube results such as the high-energy upgoing muon neutrino sample [10], from here on called $v_{\mu, u p}$, because of their limited energy range starting at a neutrino energy of around $120 \mathrm{TeV}$. This is illustrated in Fig- 


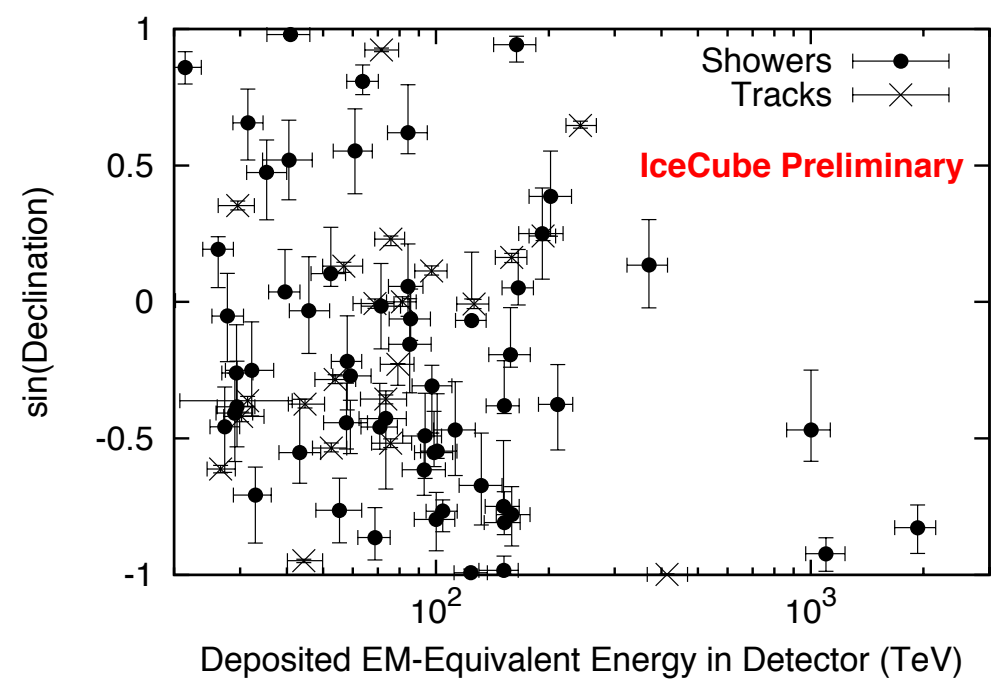

Figure 1: Arrival angles and electromagnetic-equivalent deposited energies of the events. Tracklike events are indicated with crosses whereas shower-like events are shown as filled circles. The error bars show $68 \%$ confidence intervals including statistical and systematic errors. Deposited energy as shown here is always a lower limit on the primary neutrino energy.

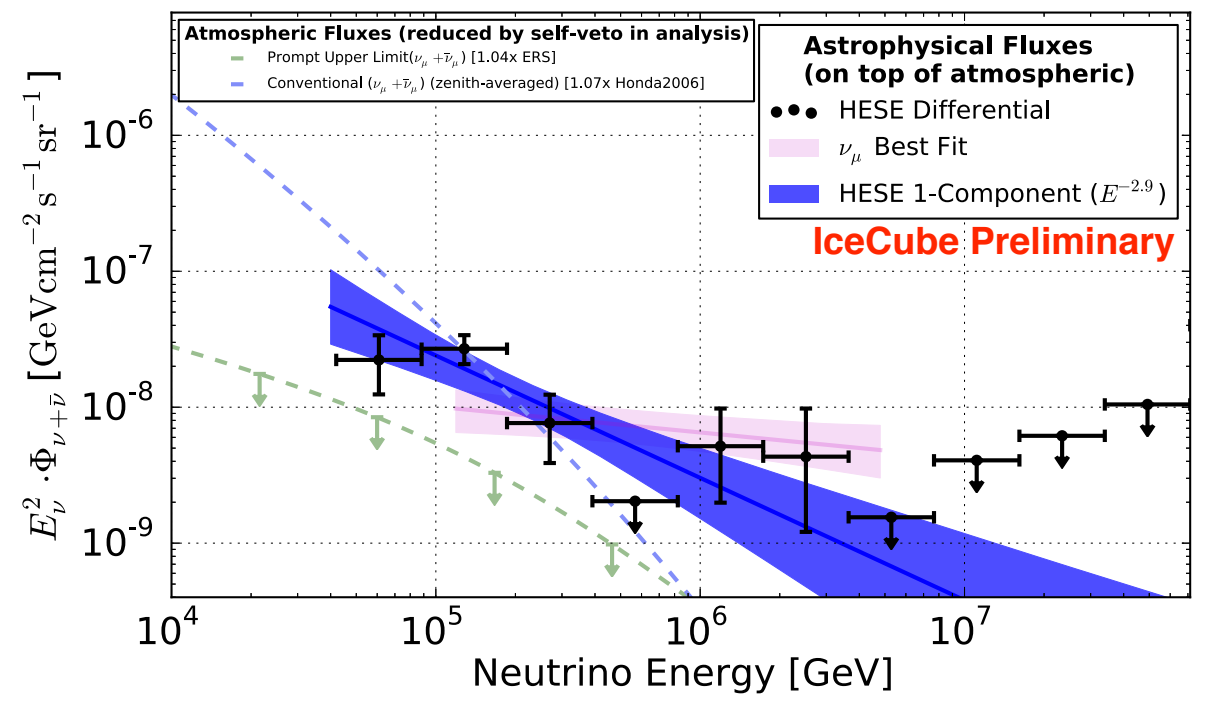

Figure 2: Best-fit per-flavor neutrino flux results (combined neutrino and anti-neutrino) as a function of energy. The black points with $1 \sigma$ uncertainties are extracted from a combined likelihood fit of all background components together with an astrophysical flux component with an independent normalization in each energy band (assuming an $E^{-2}$ spectrum within each band). The atmospheric neutrino and muon fluxes are already subtracted. The best-fit conventional flux and the best-fit upper limit on "prompt" neutrinos are shown separately, not taking into account the effect of the atmospheric self-veto, which will significantly reduce their contribution. The blue band shows the $1 \sigma$ uncertainties on the result of a single power-law fit to the HESE data. The pink band shows the $v_{\mu, u p}$ best fit [10] with $1 \sigma$ uncertainties. Its length indicates the approximate sensitive energy range of the $v_{\mu, u p}$ analysis. 
Table 1: Properties of the events observed in the fifth and sixth year. A list of events 1-37 can be found in [3], and events 38-54 in [4], respectively. The $E_{\mathrm{dep}}$ column shows the electromagneticequivalent deposited energy of each event. "Ang. Err." shows the median angular error including systematic uncertainties.

\begin{tabular}{c|c|c|c|c|c|c} 
ID & $E_{\text {dep }}(\mathrm{TeV})$ & Time (MJD) & Decl. (deg.) & R.A. (deg.) & Ang. Err. (deg.) & Topology \\
\hline 55 & --- & 56798.73029 & --- & --- & --- & Coincident \\
56 & $104.2_{-10.0}^{+9.7}$ & 56817.38958 & -50.1 & 280.5 & 6.5 & Shower \\
57 & $132.1_{-16.8}^{+18.1}$ & 56830.52665 & -42.2 & 123.0 & 14.4 & Shower \\
58 & $52.6_{-5.7}^{+5.2}$ & 56859.75882 & -32.4 & 102.1 & $<1.3$ & Track \\
59 & $124.6_{-11.7}^{+11.6}$ & 56922.58530 & -3.9 & 63.3 & 8.8 & Shower \\
60 & $93.0_{-11.7}^{+12.9}$ & 56931.93110 & -37.9 & 32.7 & 13.3 & Shower \\
61 & $53.8_{-6.3}^{+7.2}$ & 56970.20736 & -16.5 & 55.6 & $<1.2$ & Track \\
62 & $75.8_{-7.1}^{+6.7}$ & 56987.77219 & 13.3 & 187.9 & $<1.3$ & Track \\
63 & $97.4_{-9.6}^{+9.6}$ & 57000.14311 & 6.5 & 160.0 & $<1.2$ & Track \\
64 & $70.8_{-7.7}^{+8.1}$ & 57036.74378 & -27.3 & 144.5 & 10.6 & Shower \\
65 & $43.3_{-5.2}^{+5.9}$ & 57051.66378 & -33.5 & 72.8 & 17.5 & Shower \\
66 & $84.2_{-9.9}^{+10.9}$ & 57053.12727 & 38.3 & 128.7 & 18.3 & Shower \\
67 & $165.7_{-15.5}^{+16.5}$ & 57079.96532 & 3.0 & 335.7 & 7.0 & Shower \\
68 & $59.1_{-6.0}^{+8.0}$ & 57081.53526 & -15.7 & 294.3 & 11.7 & Shower \\
69 & $18.0_{-2.0}^{+2.2}$ & 57133.79007 & 0.3 & 236.2 & 15.7 & Shower \\
70 & $98.8_{-11.1}^{+12.0}$ & 57134.39812 & -33.5 & 93.9 & 12.3 & Shower \\
71 & $73.5_{-10.5}^{+10.0}$ & 57140.47276 & -20.8 & 80.7 & $<1.2$ & Track \\
72 & $35.3_{-4.1}^{+4.6}$ & 57144.29607 & 28.3 & 203.2 & 19.5 & Shower \\
73 & $26.2_{-2.3}^{+2.6}$ & 57154.83679 & 11.1 & 278.4 & 6.9 & Shower \\
74 & $71.3_{-8.1}^{+9.1}$ & 57157.00077 & -0.9 & 341.0 & 12.7 & Shower \\
75 & $164.0_{-21.4}^{+20.7}$ & 57168.40450 & 70.5 & 259.0 & 13.1 & Shower \\
76 & $126.3_{-12.7}^{+12.0}$ & 57276.56530 & -0.4 & 240.2 & $<1.2$ & Track \\
77 & $39.5_{-3.7}^{+3.8}$ & 57285.01732 & 2.1 & 278.4 & 7.2 & Shower \\
78 & $56.7_{-6.9}^{+7.0}$ & 57363.44233 & 7.5 & 0.4 & $<1.2$ & Track \\
79 & $158.2_{-19.8}^{+20.3}$ & 57365.75249 & -11.1 & 24.6 & 14.6 & Shower \\
80 & $85.6_{-10.6}^{+11.1}$ & 57386.35877 & -3.6 & 146.6 & 16.1 & Shower \\
81 & $151.8_{-21.6}^{+13.9}$ & 57480.64736 & -79.4 & 45.0 & 13.5 & Shower \\
82 & $159.3_{-15.3}^{+15.5}$ & 57505.24482 & 9.4 & 240.9 & $<1.2$ & Track \\
& & & & & &
\end{tabular}

ure 2 where we compare the $v_{\mu, u p}$ best-fit in its approximate sensitive energy range with the HESE data. The seemingly large differences in the best-fit slopes could suggest a break in the power-law spectrum arising from, e.g., a second harder astrophysical component. This possibility has been previously investigated using 4 years of HESE data [12]. Here, we performed a fit to the HESE 6-year dataset introducing a second astrophysical component, described by a power-law with an independent spectral index. A likelihood-ratio test comparing the single power-law fit with the two power-law fit gives a p-value of $37 \%$. Thus, the HESE sample is not sufficient to distinguish between these models. In a second step, we used the mostly independent ${ }^{2} v_{\mu, u p}$ best-fit astrophysical flux with $1 \sigma$ uncertainties (contour in spectral index and normalization) as a prior for the

\footnotetext{
${ }^{2}$ There is some overlap between the two samples in that some of the track-like HESE events also appear in the $v_{\mu, u p}$ selection.
} 
high-energy ("hard") component of the HESE two power-law fit. The results are shown in Fig. 3, together with the single power-law fit. A non-zero second component with a softer spectrum is then preferred by the likelihood fit. Due to the large uncertainties on this low-energy ("soft") component it is compatible with zero within about $2 \sigma$ in which case the fit reduces to a single astrophysical component. A corresponding likelihood ratio test comparing the single power-law fit with the two power-law fit using the independent $v_{\mu, u p}$ measurement as a prior yields a p-value of $1.5 \%$. Despite the strong prior, there is no clear evidence for a break in the astrophysical spectrum in the HESE data. Future IceCube analyses to be presented later this year, using samples extending to lower

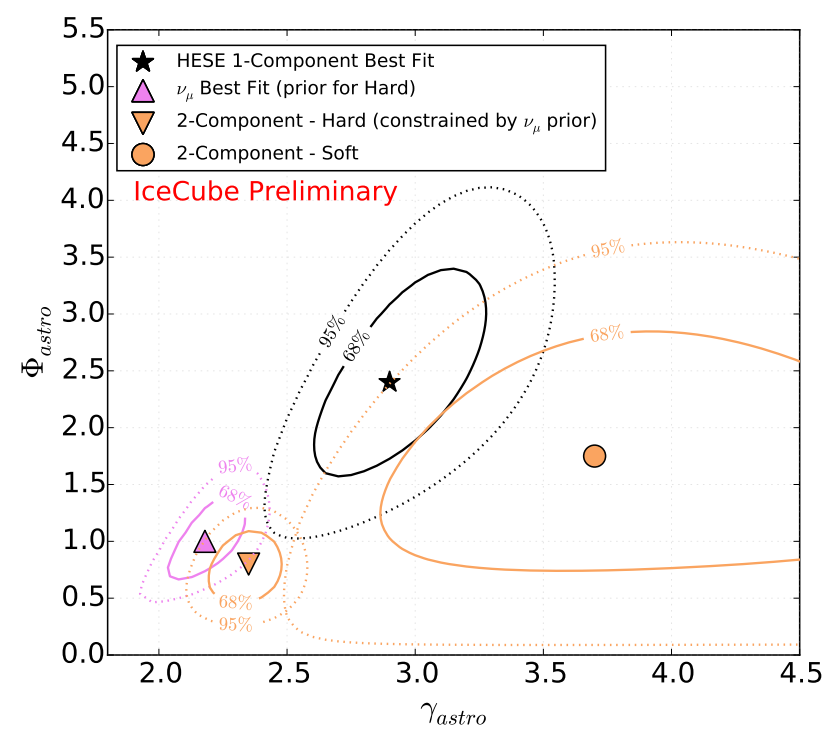

Figure 3: Contour plot of the best-fit astrophysical spectral index $\gamma_{\text {astro }}$ vs. best-fit per-flavor normalization at $100 \mathrm{TeV}, \Phi_{\text {astro }}$. Shown is the single power-law fit in black ("1-Component"), where the best-fit point is marked with a black star. The best-fit power law is $E^{2} \phi(E)=$ $2.46 \pm 0.8 \times 10^{-8}(E / 100 \mathrm{TeV})^{-0.92} \mathrm{GeVcm}^{-2} \mathrm{~s}^{-1} \mathrm{sr}^{-1}$. The orange contours show the best-fit components assuming a two power-law hypothesis with the $v_{\mu, u p}$ best fit [10], shown in pink, as a prior for the hard component. Due to the large uncertainties on the soft component it is compatible with zero within $\approx 2 \sigma$, in which case the fit reduces to a single astrophysical component.

energies and incorporating multiple channels, will have improved sensitivity to a possible break in the astrophysical spectrum. Distributions of the HESE data events compared to background and best-fit signal expectations for the above described single and two power-law model fits as functions of deposited energy and declination can be found in figures $4 \mathrm{a}$ and $4 \mathrm{~b}$, respectively.

\section{Spatial Clustering}

A maximum-likelihood clustering method [3] was used to look for any neutrino point source in the sample. The test statistic (TS) was defined as the logarithm of the ratio between the maximal likelihood including a point source component and the likelihood for the isotropic null hypothesis. The significance of our observed TS was determined by comparing to maps scrambled in right ascension. As before, the analysis was run twice, once with all events and once with only shower- 


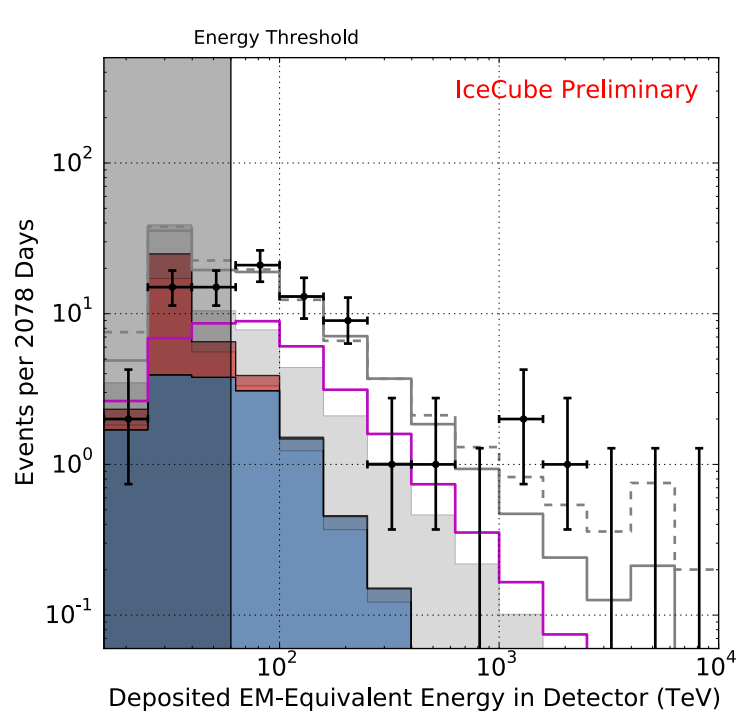

(a) deposited energies

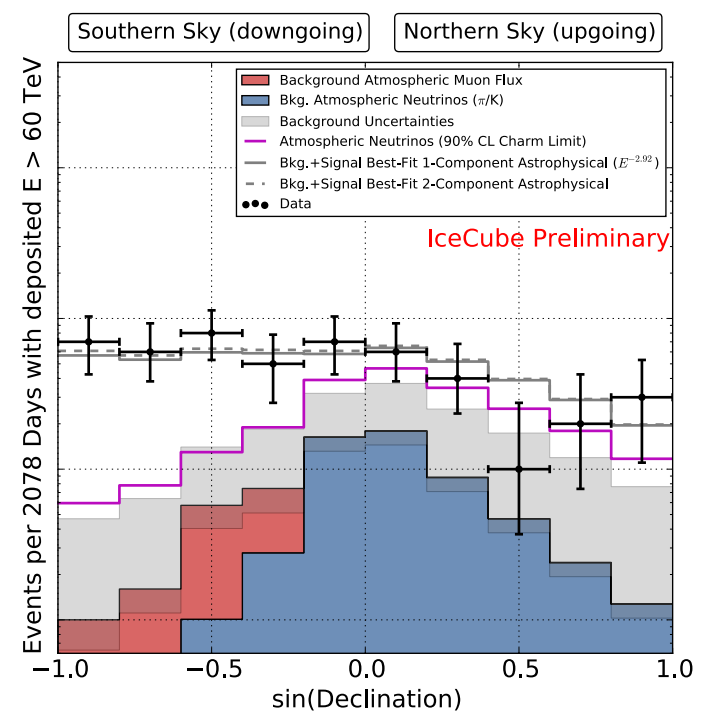

(b) arrival directions

Figure 4: Deposited energies and arrival directions of the observed events and expected contributions from backgrounds and astrophysical neutrinos. Atmospheric muon backgrounds (estimated from data) are shown in red. Atmospheric neutrino backgrounds are shown in blue with $1 \sigma$ uncertainties on the prediction shown as a gray band. For scale, the $90 \%$ CL upper bound on the charm component of atmospheric neutrinos is shown as a magenta line. The best-fit astrophysical spectra (assuming an unbroken power-law model) are shown in gray. The solid line assumes a single power-law model, whereas the dashed line assumes a two power-law model, using the spectrum derived in [10] as a prior for the high-energy component. Only events above $60 \mathrm{TeV}$ are considered in the fit.

like events in the sample. We removed events 32 and 55 (two coincident muons from unrelated air showers) and 28 (event with sub-threshold hits in the IceTop array) for purposes of all clustering analyses. This test (see Fig. 5) did not yield significant evidence of clustering with p-values of $44 \%$ and $77 \%$ for the shower-only and the all-events tests, respectively. We also performed a galactic plane clustering test using a fixed width of $2.5^{\circ}$ around the plane (p-value 23.4\%) and using a variable-width scan (p-value 17.4\%). All above p-values are corrected for trials.

\section{Future Plans}

Modified analysis strategies in IceCube have managed to reduce the energy threshold for a selection of starting events even further in order to be better able to describe the observed flux and its properties [7], but at this time they have only been applied to the first two years of data used for this study. Corresponding lower-threshold datasets, using the full set of data collected by IceCube will become available soon [11]. In addition, combined fits of this dataset and others like the through-going muon channel [10] are currently in preparation [11].

Due to the simplicity and robustness of this search with respect to systematics when compared to more detailed searches, it is well suited towards triggering and providing input for follow-up 


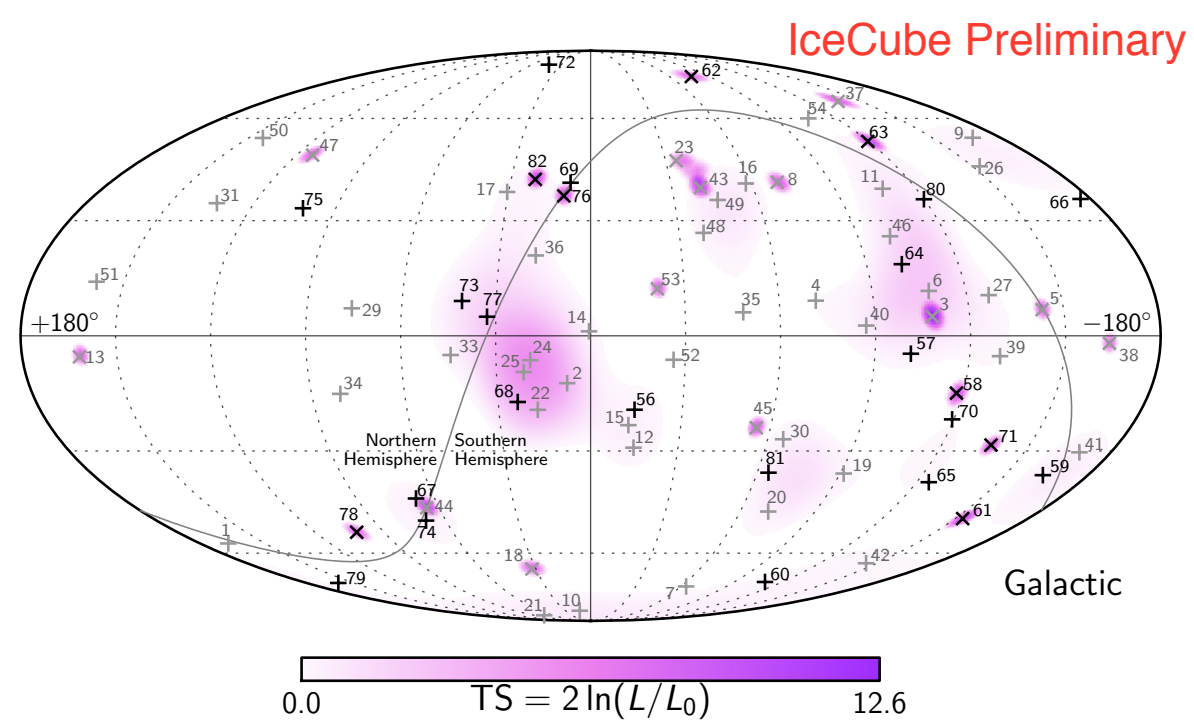

Figure 5: Arrival directions of the events in galactic coordinates. Shower-like events are marked with $\mathrm{a}+$ and those containing tracks with $\mathrm{a} \times$. The new events of table 1 are shown in black. Colors show the test statistics (TS) for the point-source clustering test at each location. No significant clustering was found.

observations by other experiments. IceCube is already sending public alerts using the HESE channel for track-like events [13] with the plan to extend this to the full HESE selection including cascade-like events soon.

\section{References}

[1] IceCube Collaboration, M. G. Aartsen et al., JINST 12 (2017) P03012

[2] IceCube Collaboration, M. G. Aartsen et al., Science 342, 1242856 (2013)

[3] IceCube Collaboration, M. G. Aartsen et al., PRL 113 (2014) 101101

[4] IceCube Collaboration, PoS ( ICRC2015) 1081 (2016)

[5] IceCube Collaboration, PoS (ICRC2017) 974 (these proceedings)

[6] IceCube Collaboration, M. G. Aartsen et al., PRD 89 (2014) 062007

[7] IceCube Collaboration, M. G. Aartsen et al., PRD 91 (2015) 022001

[8] IceCube Collaboration, M. G. Aartsen et al., ApJ 833 (2016) 1

[9] A. Cooper-Sarkar, P. Mertsch \& S. Sarkar, JHEP 08 (2011)042

[10] IceCube Collaboration, PoS (ICRC2 017) 1005 (these proceedings)

[11] IceCube Collaboration, PoS ( ICRC2017) 976 (these proceedings)

[12] A. C. Vincent, S. Palomares-Ruiz, O. Mena, PRD 94 (2016) 023009

[13] IceCube Collaboration, M. G. Aartsen et al., Astropart. Phys. 92 (2017) 30-41 